\title{
Dynamic Trading with Predictable Returns and Transaction Costs: Corrigendum
}

Equation (A15) in the appendix should be replaced

$$
A_{x x}=\bar{\Lambda}^{\frac{1}{2}}\left(\bar{\rho} \gamma \bar{\Lambda}^{-\frac{1}{2}} \Sigma \bar{\Lambda}^{-\frac{1}{2}}+\frac{1}{4}\left(\rho I+\gamma \bar{\Lambda}^{-\frac{1}{2}} \Sigma \bar{\Lambda}^{-\frac{1}{2}}\right)^{2}\right)^{\frac{1}{2}} \bar{\Lambda}^{\frac{1}{2}}-\frac{1}{2}(\rho \bar{\Lambda}+\gamma \Sigma)
$$

which follows from (A14). This expression equals (A15) when $\Sigma$ and $\Lambda$ commute (e.g., in the main case in the paper, Assumption 1), but otherwise not. 